\title{
MATHEMATICAL MODELING OF THE COUPLED PROCESSES IN NANOPOROUS BODIES
}

\author{
Taras NAHIRNYJ", Kostiantyn TCHERVINKA*
}

\author{
*Faculty of Mechanical Engineering, University of Zielona Góra, ul. prof. Z. Szafrana 4, 65-516 Zielona Góra, Poland \\ **Department of Mathematical Modeling, Ivan Franko National University of Lviv, Universytetska St., 1, Lviv, 79000, Ukraine
}

t.nahirny@iizp.uz.zgora.pl, k.tchervinka@gmail.com

received 5 April 2017, revised 18 August 2018, accepted 27 August 2018

\begin{abstract}
The methods of irreversible thermomechanics and functional analysis are used to formulate the mathematical model of thermoelastic solid body taking account of structural heterogeneity of the body material and geometric irregularity of its surface. The density and the chemical potential of skeleton among others are included into the state parameters space. The source of skeleton mass reconciles the actual and reference body states and may be associated with real surface forming method. The analysis of model problem solutions shows that the model is appropriate to describe coupled processes in porous and nanoporous bodies. It allows studying the size effects of strength, elastic moduli, etc. caused by near-surface non-homogeneity.
\end{abstract}

Key words: Continual Model, Thermoelastic Body, Coupled Processes, Nanomaterial, Size Effects

\section{INTRODUCTION}

In recent years nanomaterials have found many applications in various fields of science and technology. A number of nanomaterials have excellent mechanical, chemical, electrical or optical properties as described by Bao et al. (2016), Hu et al. (2010), Wang et al. (2009). This draws attention to the problems of modeling, simulating and calculating characteristics of the newfound materials. Among the important properties of nanomaterials one can find the size effects of elastic properties, strength, surface tension, durability, etc.

The approaches to model the mechanical properties of nanomaterials include atomistic simulation, mesoscopic modeling and enhanced continual models (Pindera et al., 2009; Rafii-Tabar et al., 2016). Some of the well-known models in the mechanics of structurally heterogeneous media for the purpose of analyzing their suitability to describe properties of nanomaterials (nanoparticles and nanocomposites) and their mechanical behavior may be found in the works by Young (2012). Among the continual models, nonlocal and gradient approaches predominate. The first approach origin goes back to works by Eringen (1972, 2002), the second one was contributed by many authors and in many variations, e.g. Maugin (1979), Polizzotto (2003), Aifantis (2011b), Geers $(2001,2002)$. Note that these approaches rarely consider processes other than mechanical, and bodies other than deformable solids. However taking account of the influence of temperature, admixtures, electric charge is important in many applications.

In the literature, the method of homogenizing is widely used to describe heterogeneous media (Charalambakis, 2010; Kachanov and Sevostianov, 2018; Markov, 2000; Kalamkarov et al., 2009). This method allows studying the effective properties of the heterogeneous material. Mechanics of periodically heterogeneous structures and homogenized models of periodic composite materials with application to thermoelasticity have been developed by Woźniak (1987) and his followers.
The local gradient approach in thermomechanics is aimed to describe structural heterogeneity and near-surface non-homogeneity in bodies with different physical fields presence. The approach originates from the study by Burak and Nahirnyj (1992) and was sufficiently reconsidered and expanded recently (Nahirnyj and Tchervinka, 2013, 2014, 2015). Its name originates from introducing gradients of state parameters into the space of thermodynamic local variables (mainly chemical potential as conjugated to the density parameter) thus increasing order and scope of classical models of solid mechanics, thermomechanics, etc.

Now the thermoelastic solid body is considered as an open thermodynamic system with variable density reflecting the structure of body and its surface (Nahirnyj and Tchervinka, 2015). The density incorporation into state parameter space and the formulated equation for it allow to describe structural heterogeneity of the material, as was shown by comparison with double diffusivity models that account for two distinct diffusivity paths throught grain and grain boundary (Nahirnyj and Tchervinka, 2012).

The models of this approach allow describing multiscale size effects of strength, elastic moduli, surface tension (other approaches to model such phenomena may be found in works by Dönmez and Bažant, 2017; Elliott, 2011). We interpret the nonhomogeneity in the density distribution and its difference from initial value as a variable porosity of structurally heterogeneous material for continual description. Hence the models of local gradient approach in thermomechanics allow describing the size effects, different physical nature fields effects on stress-strain state and variable density inherent to the structured e.g. porous body. We also note that this approach to the description of porosity is fundamentally different from other well-known approaches (Bio, 1941; Coussy, 2004; Vafai, 2015).

The mass sources introduced into the model of one-component body allow, in particular, to take into account a geometric non-uniformity of the real bodies surface, i.e. its roughness, waviness. The above factors (namely, the heterogeneity of the materi- 
al and mass sources) are the causes of the non-homogeneity of considered fields in the bodies, that are free from the external force load.

In this paper, the key relations of local gradient approach, its applications to nanoporous solid solution description and some model problems solution analysis are presented .

\section{MODEL APPROACH}

We consider a two-component thermoelastic body consisting of skeleton and admixture. The considered processes are deformation, diffusion and thermal conductivity. According to the general principles of irreversible thermodynamics and theory of rheological systems we shall formulate balance equations for energy and additive quantity of every form of motion within the considered processes. The body is considered as an open thermodynamic system whose mass changes relatively to the uniform reference body containing no admixture. The material structure and body surface have arisen at the initial moment of time $\tau=0$ and do not change afterward $(\tau>0)$. The flux of skeleton mass $\boldsymbol{j}_{m 0}$ and mass sources $\sigma_{m 0}$ are the model parameters used to describe the process of the body structure forming (see Fig.1). The admixture with the density $\varrho_{1}$ and the flux $\boldsymbol{j}_{m 1}$ is considered according to the classical theory of diffusion.

\subsection{Balance equations for solid solution}

The local balance equations for momentum $\boldsymbol{k}_{v}$, entropy $S$, masses of skeleton and admixture have the form:

$\frac{\partial \boldsymbol{k}_{v}}{\partial \tau}=\boldsymbol{\nabla} \cdot \boldsymbol{\sigma}, \quad \frac{\partial S}{\partial \tau}=-\boldsymbol{\nabla} \cdot \boldsymbol{j}_{S}+\sigma_{s}$,

$\frac{\partial \varrho_{0}}{\partial \tau}=-\boldsymbol{\nabla} \cdot \boldsymbol{j}_{m 0}+\sigma_{m 0}, \frac{\partial \varrho_{1}}{\partial \tau}=-\boldsymbol{\nabla} \cdot \boldsymbol{j}_{m 1}$.

Here $\boldsymbol{\sigma}$ is the Cauchy stress tensor, $\boldsymbol{j}_{s}$ and $\sigma_{s}$ are the entropy flux and its production, respectively, $\boldsymbol{\nabla}$ is the del operator.

The local form of the balance equation for total energy $E=U+K$ (where $K$ and $U$ are the kinetic and internal energies, respectively) are written as follows:

$\frac{\partial(U+K)}{\partial \tau}=\boldsymbol{\nabla} \cdot\left(\boldsymbol{\sigma} \cdot \boldsymbol{v}-T \boldsymbol{j}_{S}-H_{0} \boldsymbol{j}_{m 0}-H_{1} \boldsymbol{j}_{m 1}\right)+\sigma_{E}$.

Here $\boldsymbol{v}$ is velocity vector, $T, H_{0}, H_{1}$ are temperature, chemical potentials of skeleton and admixture, $\sigma_{E}$ is an energy source related to body structure formation. We take that $\sigma_{m 0}=\sigma_{E} / H_{0}$.

We assume that $d K=\boldsymbol{v} \cdot d \boldsymbol{k}_{v}$ and from (1),(2) obtain the following equation for the internal energy:

$\frac{\partial U}{\partial \tau}=\boldsymbol{\sigma}: \frac{\partial \boldsymbol{e}}{\partial \tau}+T \frac{\partial S}{\partial \tau}+H_{0} \frac{\partial \varrho_{0}}{\partial \tau}+H_{1} \frac{\partial \varrho_{1}}{\partial \tau}$

$-T \sigma_{s}-\boldsymbol{j}_{s} \cdot \boldsymbol{\nabla} T-\boldsymbol{j}_{m 0} \cdot \boldsymbol{\nabla} H_{0}-\boldsymbol{j}_{m 1} \cdot \boldsymbol{\nabla} H_{1}$

Here $\boldsymbol{e}$ is the strain tensor, the colon ":" represent double inner product.

Eq. (3) that is sometimes referred to as the internal energy balance equation is the basis for formulating constitutive relations of the thermodynamical model. The key point of local gradient approach is the proper choice of kinetic relations (Burak et al., 1992).

Note that some other approaches also use modification of kinetic relations in modeling processes in heterogeneous media. For instance, the kinetic relations for phase transition fronts and straight through crack propagation are derived on the basis of the material description of continuum mechanics and the thermodynamics of discrete systems by Berezovski et al. (2007); unstable kinetic relations with application to phase transitions are considered by Rosakis and Knowles, (1997); the viscosity and strain-gradient effects are considered also by Abeyaratne and Knowles, (1991).

\subsection{Constitutive relation for solid solution}

Within the linear approach the linear functions of thermodynamic forces $\boldsymbol{\nabla} T, \boldsymbol{\nabla} H_{0}, \boldsymbol{\nabla} H_{1}$ for the fluxs $\boldsymbol{j}_{s}, \boldsymbol{j}_{m 0}, \boldsymbol{j}_{m 1}$ are conventionally accepted. However we assume that skeleton mass flux contains a term describing the non-fading memory of body structure formation:

$\boldsymbol{j}_{m 0}=\boldsymbol{j}_{m c}+\boldsymbol{j}_{m h}, \quad \boldsymbol{j}_{m h}=g_{m m} \frac{\partial\left(\nabla H_{0}\right)}{\partial \tau}$.

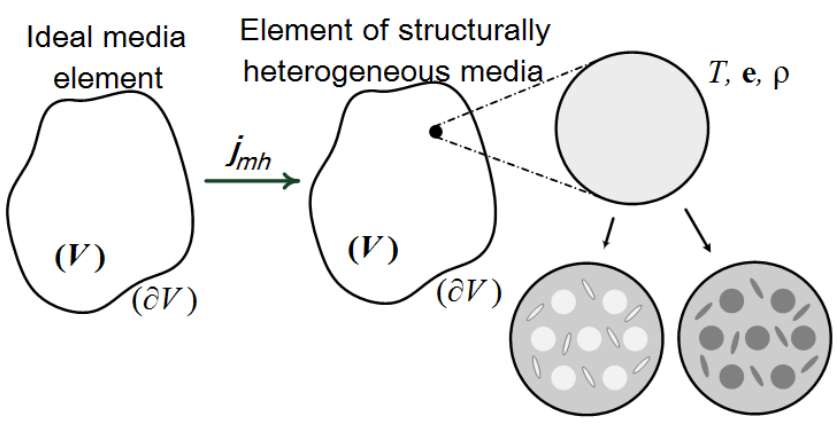

Fig. 1. The ideal medium vs. structurally heterogeneous medium and its physically small element

According to the theory of hereditary media, the flux is represented as integral over the history and the non-fading memory is described by term with singular kernel (Rabotnov, 1980) resulting in the flux dependence on the time derivative of the force.

The other kinetic relations are:

$\boldsymbol{j}_{m c}=-g_{m s 0} \boldsymbol{\nabla} T-g_{00} \nabla H_{0}-g_{01} \nabla H_{1}$,

$\boldsymbol{j}_{m 1}=-g_{m s 1} \boldsymbol{\nabla} T-g_{10} \boldsymbol{\nabla} H_{0}-g_{11} \nabla H_{1}$,

$\boldsymbol{j}_{S}=-\lambda_{s s} \frac{\nabla T}{T}-\lambda_{s m 0} \frac{\nabla H_{0}}{T}-\lambda_{s m 1} \frac{\nabla H_{1}}{T}$.

Here $g_{i j}, \lambda_{i j}$ are constants, hereinafter indices $i, j$ run through all values present in the above formula.

From Eq. (3) using the above kinetic representation and the expression for the dissipation energy:

$T \sigma_{s}=-\boldsymbol{j}_{s} \cdot \boldsymbol{\nabla} T-\boldsymbol{j}_{m c} \cdot \boldsymbol{\nabla} H_{0}-\boldsymbol{j}_{m 1} \cdot \boldsymbol{\nabla} H_{1}$,

we obtain

$\frac{\partial}{\partial \tau}\left(U-\frac{g_{m m}}{2}\left(\boldsymbol{\nabla} H_{0}\right) \cdot\left(\boldsymbol{\nabla} H_{0}\right)\right)$

$=\boldsymbol{\sigma}: \frac{\partial \boldsymbol{e}}{\partial \tau}+T \frac{\partial S}{\partial \tau}+H_{0} \frac{\partial \varrho_{0}}{\partial \tau}+H_{1} \frac{\partial \varrho_{1}}{\partial \tau}$. formula:

Introducing the free energy $F$ of heterogeneous material with

$F=U-\frac{g_{m m}}{2}\left(\boldsymbol{\nabla} H_{0}\right) \cdot\left(\boldsymbol{\nabla} H_{0}\right)-T S$

from (7) we obtain the Gibbs equation: 
$d F=\boldsymbol{\sigma}: d \boldsymbol{e}-S d T+H_{0} d \varrho_{0}+H_{1} d \varrho_{1}$

$\frac{\partial}{\partial \tau}\left(\varrho_{0}+\varrho_{1}+g_{m m} \nabla^{2} H_{0}\right)=\sigma_{m 0}$.

and write the state equations:

$\boldsymbol{\sigma}=\frac{\partial F}{\partial \boldsymbol{e}}, S=-\frac{\partial F}{\partial T}, H_{0}=\frac{\partial F}{\partial \varrho_{0}}, H_{1}=\frac{\partial F}{\partial \varrho_{1}}$

The energy $F$ is defined on the parameter space that contains the strain, the temperature and the densities of skeleton and admixture $\boldsymbol{e}, T, \varrho_{0}, \varrho_{1}$. The conjugated parameters are the stress, the entropy and the chemical potentials of skeleton and admixture $\boldsymbol{\sigma}, S, H_{0}, H_{1}$.

The key equation system contains the balance equations (1), the kinetic relations (4), (5), the state equations (10) and some other relations, i.e. expression (6), strain-deformation relation $2 \boldsymbol{e}=\boldsymbol{\nabla} \otimes \boldsymbol{u}+(\boldsymbol{\nabla} \otimes \boldsymbol{u})^{T}(\otimes$ is dyadic product, superscript $T$ denotes transposition), expression for the translational momentum $\boldsymbol{k}_{v}=\left(\varrho_{0}+\varrho_{1}\right) \frac{\partial \boldsymbol{u}}{\partial \tau}$. To write solving equation system we need to use some representation of the energy $F\left(e, T, \varrho_{0}, \varrho_{1}\right)$. The Taylor series representation in the vicinity of a reference state is usually used.

We consider the reference state in which:

$\boldsymbol{e}=\mathbf{0}, T=T_{*}, \varrho_{0}=\varrho_{0 *}, \varrho_{1}=\varrho_{1 *}=0$,

$\boldsymbol{\sigma}=\mathbf{0}, S=S_{*}, H_{0}=H_{0 *}, H_{1}=H_{1 *}$

and denote $\theta=T-T_{*}$. Then the quadratic representation of the energy $F\left(\boldsymbol{e}, \theta, \varrho_{0}, \varrho_{1}\right)$ written as

$F=F_{*}-S_{*} \theta+H_{0 *}\left(\varrho_{0}-\varrho_{0 *}\right)+H_{1 *} \rho_{1}+\mu \boldsymbol{e}: \boldsymbol{e}-a_{e t} e \theta$

$+\frac{\lambda}{2} e^{2}-\frac{1}{2} a_{t t} \theta^{2}-a_{e m 0} e\left(\varrho_{0}-\varrho_{0 *}\right)-a_{e m 1} e \varrho_{1}$

$-a_{t m 0} \theta\left(\varrho_{0}-\varrho_{0 *}\right)-a_{t m 1} \theta \rho_{1}+a_{m 0 m 1}\left(\varrho_{0}-\varrho_{0 *}\right) \varrho_{1}$

$+\frac{1}{2} a_{m 0 m 0}\left(\varrho_{0}-\varrho_{0 *}\right)^{2}+\frac{1}{2} a_{m 1 m 1} \varrho_{1}^{2}$

leads to the linear equations of state:

$\boldsymbol{\sigma}=2 \mu \boldsymbol{e}+\left[\lambda e-a_{e m 0}\left(\varrho_{0}-\varrho_{0 *}\right)-a_{e m 1} \varrho_{1}-a_{e t} \theta\right] \boldsymbol{I}$,

$S=S_{*}+a_{e t} e+a_{t m 0}\left(\varrho_{0}-\varrho_{0 *}\right)+a_{t m 1} \varrho_{1}+a_{t t} \theta$,

$H_{0}=H_{0 *}-a_{e m 0} e+a_{m 0 m 0}\left(\varrho_{0}-\varrho_{0 *}\right)$

$+a_{m 0 m 1} \varrho_{1}-a_{t m 0} \theta$,

$H_{1}=H_{1 *}-a_{e m 1} e+a_{m 0 m 1}\left(\varrho_{0}-\varrho_{0 *}\right)$

$+a_{m 1 m 1} \varrho_{1}-a_{t m 1} \theta$.

Here $\boldsymbol{I}$ is the identity tensor, $e=\boldsymbol{e}: \boldsymbol{I}=\boldsymbol{\nabla} \cdot \boldsymbol{u}, \lambda, \mu, a_{i j}$ are the constants.

\subsection{Key equations systems}

Note that representation (4) modifies the third equation of (1) to the form:

$\frac{\partial \varrho_{0}}{\partial \tau}+g_{m m} \boldsymbol{\nabla} \cdot \frac{\partial\left(\boldsymbol{\nabla} H_{0}\right)}{\partial \tau}=-\boldsymbol{\nabla} \cdot \boldsymbol{j}_{m c}+\sigma_{m 0}$.

In the study of solid solutions along with the systems of skeleton and admixtures it is convenient to consider the continuum of mass centers for which the total mass flux is zero. Taking this flux as $\boldsymbol{j}_{m c}+\boldsymbol{j}_{m 1}=\mathbf{0}$ we write the equation of mass balance as follows:
By integrating this equation over time we obtain:

$\varrho_{0}-\varrho_{0 *}+\varrho_{1}+g_{m m} \nabla^{2} H_{0}=d_{\sigma m}$.

Here the relations $\varrho_{0}=\varrho_{0 *}, \varrho_{1}=\varrho_{1 *}=0$ and $H_{0 *}=$ const for reference state have been used along with notation

$d_{\sigma m}=\int_{0}^{\tau} \sigma_{m 0} d \tau$.

An extra integral condition is needed to reconcile the actual and reference state. The supply from mass source must be equal to difference between masses of reference body of density $\varrho_{0 \text { * }}$ and real body (without admixtures) of density $\varrho_{0}$ :

$\int_{(V)} d_{\sigma m} d V=\int_{(V)}\left(\varrho_{0}-\varrho_{0 *}\right) d V$.

If $g_{m m}=0$, then (13) turns into:

$\varrho_{0}-\varrho_{0 *}+\varrho_{1}=d_{\sigma m}$

and we deal with porous body. In the absence of admixture the density $\varrho_{0}$ is different from the reference body density $\varrho_{0 *}$ in addend introduced above with mass source $\sigma_{m 0}$ :

$\varrho_{0}=\varrho_{0 *}+d_{\sigma m}$.

In the case $g_{m m} \neq 0$ the body may be treated as nanoporous body. This, in particular, is confirmed by comparing formula (13) with the results presented in the work of Aifantis (2011a) as was done by Nahirnyj and Tchervinka (2012).

Eq. (13) contains the laplacians of the first strain invariant $e$, the densities $\varrho_{0}, \varrho_{1}$, the temperature $\theta$ (see third equation of (11)). In the stationary state they may be eliminated from Eq. (13) using the others equations of the system. The first equations of (1) and (11) in static and quasistatic cases yield:

$\boldsymbol{\nabla} \cdot \boldsymbol{\sigma}=0, \quad \Rightarrow$

$(\lambda+2 \mu) \nabla^{2}(\boldsymbol{\nabla} \cdot \boldsymbol{u})-a_{e t} \nabla^{2} \theta$

$-a_{e m 0} \nabla^{2}\left(\varrho_{0}-\varrho_{0 *}\right)-a_{e m 1} \nabla^{2} \varrho_{1}=0, \quad \Rightarrow$

$\nabla^{2}(\boldsymbol{\nabla} \cdot \boldsymbol{u})=\frac{a_{e t}}{\lambda+2 \mu} \nabla^{2} \theta+\frac{a_{e m 0}}{\lambda+2 \mu} \nabla^{2}\left(\varrho_{0}-\varrho_{0 *}\right)+\frac{a_{e m 1}}{\lambda+2 \mu} \nabla^{2} \varrho_{1}$.

This relation allows to exclude the term $\nabla^{2} e$, and from heat conduction equation in stationary case it follows that $\nabla^{2} \theta=0$. Thus the mass balance equation for skeleton leads to non-homogeneous Helmholtz equation:

$-\xi^{-2} \nabla^{2} \varrho_{0}+\varrho_{0}-\varrho_{0 *}+\varrho_{1}=d_{\sigma m}$,

where $\xi$ is the constant.

In general case the solving equation system is written in terms of displacement, temperature, densities of admixture and skeleton using the standard linearization technique in heat conduction equation:

$\mu \nabla^{2} \boldsymbol{u}+(\lambda+\mu) \boldsymbol{\nabla}(\boldsymbol{\nabla} \cdot \boldsymbol{u})-a_{e t} \boldsymbol{\nabla} \theta$

$-a_{e m 0} \nabla\left(\varrho_{0}-\varrho_{0 *}\right)-a_{e m 1} \nabla \rho_{1}=\frac{\partial}{\partial \tau}\left[\left(\varrho_{0}+\varrho_{1}\right) \frac{\partial u}{\partial \tau}\right]$,

$a_{t t} T_{*} \frac{\partial \theta}{\partial \tau}+a_{e t} T_{*} \frac{\partial(\boldsymbol{\nabla} \cdot \boldsymbol{u})}{\partial \tau}+a_{t m 0} T_{*} \frac{\partial \varrho_{0}}{\partial \tau}+a_{t m 1} T_{*} \frac{\partial \varrho_{1}}{\partial \tau}$

$=\gamma_{t t} \nabla^{2} \theta+\gamma_{t m 0} \nabla^{2} \varrho_{0}+\lambda_{t m 1} \nabla^{2} \varrho_{1}$,

$\frac{\partial \varrho_{1}}{\partial \tau}=\gamma_{m 1 t} \nabla^{2} \theta+\gamma_{t m 0} \nabla^{2} \varrho_{0}+\lambda_{t m 1} \nabla^{2} \varrho_{1}$,

$\gamma_{t} \nabla^{2} \theta+\gamma_{e} \nabla^{2} e+\gamma_{m 0} \nabla^{2} \varrho_{0}+\gamma_{m 1} \nabla^{2} \varrho_{1}$

$+\varrho_{0}-\varrho_{0 *}+\varrho_{1}=d_{\sigma m}$. 
Here $\gamma_{i}, \gamma_{i j}$ are the constants.

If we assume that $T=T_{*}$ and confine ourselves to isothermal and static case, the key system of equations takes the form:

$-\xi^{-2} \nabla^{2} \varrho_{0}+\varrho_{0}-\varrho_{0 *}+\varrho_{1}=d_{\sigma m}$

$a \nabla^{2} \varrho_{0}+\nabla^{2} \varrho_{1}=0$

$\mu \nabla^{2} \boldsymbol{u}+(\lambda+\mu) \boldsymbol{\nabla}(\boldsymbol{\nabla} \cdot \boldsymbol{u})-a_{e t} \boldsymbol{\nabla} \theta$

$-a_{e m 0} \nabla\left(\varrho_{0}-\varrho_{0 *}\right)-a_{e m 1} \nabla \varrho_{1}=0$,

where $a, \xi$ are constants.

If instead of the displacement $\boldsymbol{u}$ the stress tensor is chosen as a key function, Eq. (17) is replaced by two equations:

$\boldsymbol{\nabla} \cdot \boldsymbol{\sigma}=0$,

$\boldsymbol{\nabla} \times\left[\boldsymbol{\sigma}-\frac{2 \mu}{3 \lambda+2 \mu}\left(\frac{\lambda}{2 \mu} \sigma-a_{e m 0} \varrho_{0}-a_{e m 1} \varrho_{1}\right) \mathbf{I}\right] \times \boldsymbol{\nabla}=0$.

When the skeleton density is known the admixture distribution, temperature and stresses or displacements are found using classical methods of thermomechanics.

The boundary value problem for the skeleton density needs boundary condition $\left.\varrho_{0}\right|_{\Sigma}=\varrho_{0 a}$ at the body surface $\Sigma$ that was discussed by Nahirnyj and Tchervinka (2015) where the significance of the surface roughness for boundary condition formulation was indicated.

\subsection{Linear and nonlinear model formulation}

If coefficients in kinetic relations (4),(5) and equations of state (11) are constant, the solving systems (16), (17) and (16), (18) are linear. The system (15) becomes linear if the density in expression for momentum is taken as $\varrho_{0}+\varrho_{1} \approx \varrho_{0 *}$. However there is a strong argument to consider nonlinear formulation.

One of the model parameters is the density. In porous and nanoporous bodies the density varies strongly. When modeling surface roughness influence on mechanical properties of elements, one considers surface value of density to be half of the bulk value (Nahirnyj et al., 2015), in metal foams the density may be one tenth and less (Bhattacharya et al., 2002; Tappan et al., 2010). The density of porous material depends directly on porosity coefficient $\phi$ and so the elastic properties depend on it. The Young's modulus and Poisson's ratio are often expressed as a power of $(1-\phi)$. So the coefficients state equation (11) (and in energy $F$ representation) may be considered as dependent on density:

$\lambda=\lambda\left(\varrho_{0}, \varrho_{1}\right), \mu=\mu\left(\varrho_{0}, \varrho_{1}\right), a_{i j}=a_{i j}\left(\varrho_{0}, \varrho_{1}\right)$.

Alternatively the higher terms in energy $F$ series expansion may be kept that leads to nonlinear state equations. The onedimensional problem in Cartesian coordinates for the thermoelastic thin film was solved analytically (Nahirnyj et al., 2015). The solution of the boundary value problem for a stretched layer was used to study the size effect of the effective Young's modulus in the body without admixture.

The linear problem formulation is considered below on example on the thermoelastic layer without admixture.

\subsection{Thermoelastic layer}

We consider a thermoelastic one-component layer $|x| \leq l$ in Cartesian coordinates $\{x, y, z\}$. The layer is stretched at infinity $y \rightarrow \pm \infty$ with force $\boldsymbol{F}=\left(0,2 l \sigma_{a}, 0\right)$ and the constant value of density $\varrho_{0}=\varrho_{0 a}>0$ is set at surfaces $x= \pm l$. The solving functions of key system (15),(18) depend on variable $x$ only.

$\frac{d^{2} \rho_{0}}{d x^{2}}-\xi^{2}\left(\rho_{0}-\rho_{0 *}\right)=-\xi^{2} d_{\sigma m}, \quad \frac{d^{2} \theta}{d x^{2}}=0$,

$\frac{d \sigma_{x x}}{d x}=0, \quad \frac{d^{2} \sigma_{y y}}{d x^{2}}=\frac{d^{2} \sigma_{z z}}{d x^{2}}=-a_{0} \frac{d^{2} \rho_{0}}{d x^{2}}$

where $a_{0}=\frac{2 \mu a_{e m 0}}{\lambda+2 \mu}$.

We specify the following boundary conditions:

$\rho_{0}=\rho_{0 a}, \quad \theta=\theta_{a}, \quad \sigma_{x x}=0$

at $x= \pm l$ and integral conditions for stresses:

$\int_{-l}^{l} \sigma_{y y} d x=2 l \sigma_{a}, \int_{-l}^{l} x \sigma_{y y} d x=0$,

$\int_{-l}^{l} \sigma_{z z} d x=0, \int_{-l}^{l} x \sigma_{z z} d x=0$.

The condition (14) for density must be satisfied as well:

$\int_{-l}^{l} d_{\sigma m} d x=\int_{-l}^{l}\left(\varrho_{0}-\varrho_{0 *}\right) d x$.

Assuming the mass sources to decrease exponentially with distance from surfaces, using the problem symmetry and the condition (22) we obtain:

$d_{\sigma m}=\left(\rho_{0 a}-\rho_{0 *}\right)\left(\frac{\vartheta^{2}}{\xi^{2}}-1\right) \frac{D}{1-D} \frac{\cosh (\vartheta x)}{\cosh (\vartheta l)}$,

$D=\frac{\xi}{\vartheta} \frac{\tanh (\xi l)}{\tanh (\vartheta l)}$

The solution of the problem (19)-(23) is

$\theta(x)=\theta_{a}$,

$\varrho_{0}(x)=\varrho_{0 a}+\frac{\varrho_{0 a}-\varrho_{0 *}}{1-D}\left(\frac{\cosh (\xi x)}{\cosh (\xi l)}-1\right)$

$-\frac{\varrho_{0 a}-\varrho_{0 *}}{1-D} D\left(\frac{\cosh (\vartheta x)}{\cosh (\vartheta l)}-1\right)$,

$\sigma_{y y}(x)=\sigma_{a}-a_{0}\left[\frac{\varrho_{0 a}-\varrho_{0 *}}{1-D}\left(\frac{\cosh (\xi x)}{\cosh (\xi l)}-1\right)\right.$

$\left.-\frac{\varrho_{0 a}-\varrho_{0 *}}{1-D} D\left(\frac{\cosh (\vartheta x)}{\cosh (\vartheta l)}-1\right)\right]$,

$\sigma_{z z}(x)=\sigma_{y y}(x)-\sigma_{a}, \quad \sigma_{x x}(x)=0$.

If the layer is free of load $\left(\sigma_{a}=0\right)$ then there is the stresses proportional to density disturbance $\varrho_{0 a}-\varrho_{0 *}$. The density along with thermal load $\theta_{a}$ also affect strain component $e_{x x}$. From the first equation of (11) one obtains:

$\boldsymbol{e}=\frac{\boldsymbol{\sigma}}{2 \mu}-\frac{\lambda \sigma \boldsymbol{I}}{2 \mu(3 \lambda+2 \mu)}+\frac{a_{e m}\left(\varrho_{0}-\varrho_{0 *}\right) \boldsymbol{I}}{3 \lambda+2 \mu}+\frac{a_{e t} \theta \boldsymbol{I}}{3 \lambda+2 \mu}$.

or using Young's modulus and Poisson's ratio:

$\boldsymbol{e}=\frac{1+v}{E} \boldsymbol{\sigma}-\frac{v}{E} \sigma \boldsymbol{I}+\frac{1-2 v}{E}\left(a_{e m}\left(\varrho_{0}-\varrho_{0 *}\right)+a_{e t} \theta\right) \boldsymbol{I}$.

From solution (24) and relation (25) we obtain:

$e_{x x}=\frac{-\lambda \sigma_{a}}{2 \mu(3 \lambda+2 \mu)}+\frac{a_{e t} \theta}{3 \lambda+2 \mu}+\frac{a_{e m}}{3 \lambda+2 \mu}\left[\frac{\varrho_{0 a}-\varrho_{0 *}}{1-D}\left(\frac{\cosh (\xi x)}{\cosh (\xi l)}-1\right)\right.$

$\left.-\frac{\varrho_{0 a}-\varrho_{0 *}}{1-D} D\left(\frac{\cosh (\vartheta x)}{\cosh (\vartheta l)}-1\right)\right]$

$e_{y y}=\frac{(\lambda+\mu) \sigma_{a}}{\mu(3 \lambda+2 \mu)}+\frac{a_{e t} \theta}{3 \lambda+2 \mu}$

$e_{z z}=-\frac{\lambda \sigma_{a}}{2 \mu(3 \lambda+2 \mu)}+\frac{a_{e t} \theta}{3 \lambda+2 \mu}$. 
Thus for the formulated problem the non-homogeneity causes deformation only toward body surfaces.

\section{MODELING OF POROUS AND NANOPOROUS BODY}

Within the framework of the model, the density of the body can vary spatially. It is naturally to take that the elastic moduli present in equations of state must account for these changes. This applies to porous materials, hence we consider

$E=E_{0}\left(\frac{\varrho_{0}}{\varrho_{0 *}}\right)^{\beta_{E}}, v=v_{0}\left(\frac{\varrho_{0}}{\varrho_{0 *}}\right)^{\beta_{N}}$

for local Young's modulus and Poisson's ratio $\left(E_{0}, v_{0}\right.$ are the moduli of bulk material, $\beta_{E}, \beta_{N}$ are constants).

The parameters $\lambda, \mu$ in (11) depend on the density through relations:

$\lambda=\frac{v E}{(1+v)(1-2 v)}, \mu=\frac{E}{2(1+v)}$.

The obtained nonlinear equation systems (16), (17) and (16), (18) describe distribution of stresses/deformation, temperature and admixtures of skeleton and admixture in the bodies of structurally heterogeneous material and with rough surface.

The relations (26) describe the elastic properties in every point of considered body. If deformation of entire body is considered, the effective Young's modulus and Poisson's ratio may be found. In paper by Nahirnyj et al. (2015) the stretched elastic layer $|x| \leq l$ was examined and the effective Young's modulus dependence on the layer size was found. It is plotted in Fig.2. Note that in the case of homogeneous body $E=E_{0}, v=v_{0}$.

Taking into account the local elastic moduli depending on the density is important in terms of describing the behavior of nanoelements. Density and elasticity moduli are equally important material characteristics.

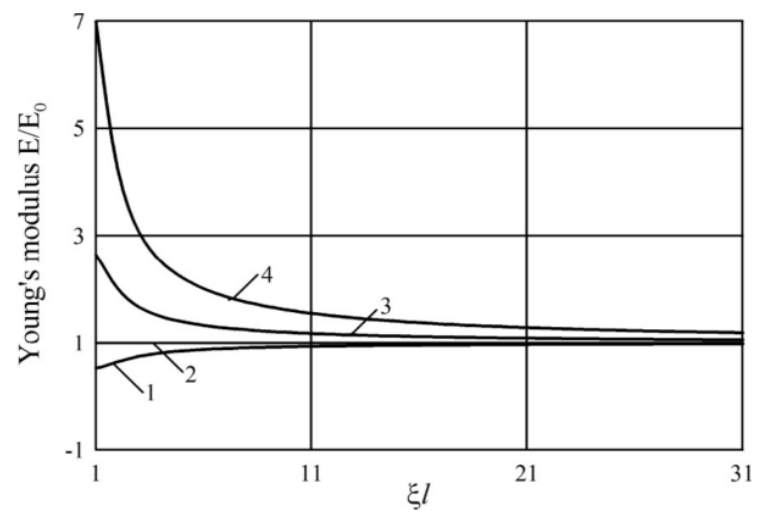

Fig. 2. The effective Young's modulus dependence on the layer thickness $(\xi l)$ (size effect of effective Young's modulus) for various values of parameter $\beta_{E}$ in $(26)\left(\beta_{E}=1 ; 0 ;-1.5 ;-3\right.$ curves $1-4$ respectively)

Now we consider a one-dimensional problem for the layer saturated with the admixture using the key equation system (16), (18) in linear formulation.

\subsection{The two-component layer}

We now consider a layer $|x| \leq l$ in Cartesian coordinates $\{x, y, z\}$ at initial temperature $T=T_{*}$. The layer is stretched at infinity $y \rightarrow \pm \infty$ with force $\boldsymbol{F}=\left(0,2 l \sigma_{a}, 0\right)$ and the constant non-zero values of densities $\varrho_{0}=\varrho_{0 a}, \varrho_{1}=\varrho_{1 a}$, are set at surfaces $x= \pm l$. The key functions in system (16),(18) depend on variable $x$ and the system has the form:

$\frac{d^{2} \rho_{0}}{d x^{2}}-\xi^{2}\left(\rho_{0}-\rho_{0 *}+\rho_{1}\right)=-\xi^{2} d_{\sigma m}$

$a \frac{d^{2} \rho_{0}}{d x^{2}}+\frac{d^{2} \rho_{1}}{d x^{2}}=0$,

$\frac{d \sigma_{x x}}{d x}=0, \frac{d^{2} \sigma_{y y}}{d x^{2}}=\frac{d^{2} \sigma_{z z}}{d x^{2}}=-a_{0} \frac{d^{2} \rho_{0}}{d x^{2}}-a_{1} \frac{d^{2} \rho_{1}}{d x^{2}}$,

where $a_{i}=\frac{2 \mu a_{e m i}}{\lambda+2 \mu}, i=1,2$.

We also assume that relations (23) and conditions (21),(22) are held along with the conditions at layer surfaces:

$\rho_{0}=\rho_{0 a}, \quad \rho_{1}=\rho_{1 a}, \quad \sigma_{x x}=0$.

The solution of the model problem is

$\varrho_{0}=\varrho_{0 a}+\frac{\varrho_{0 a}-\varrho_{0 *}+\varrho_{1 a}}{1-a}\left(\frac{\cosh (\vartheta x)}{\cosh (\vartheta l)}-1\right)$

$+m_{s} \frac{\xi^{2}}{\zeta^{2}-\vartheta^{2}}\left(\frac{\cosh (\vartheta x)}{\cosh (\vartheta l)}-\frac{\cosh (\zeta x)}{\cosh (\zeta l)}\right)$,

$\varrho_{1}(x)=\varrho_{1 a}-a \frac{\varrho_{0 a}-\varrho_{0 *}+\varrho_{1 a}}{1-a}\left(\frac{\cosh (\vartheta x)}{\cosh (\vartheta l)}-1\right)$

$-a m_{s} \frac{\xi^{2}}{\zeta^{2}-\vartheta^{2}}\left(\frac{\cosh (\vartheta x)}{\cosh (\vartheta l)}-\frac{\cosh (\zeta x)}{\cosh (\zeta x)}\right)$,

$\sigma_{y y}(x)=\sigma_{a}+\left(a a_{1}-a_{0}\right)\left[\left(\frac{\varrho_{0 a}-\varrho_{0 *}+\varrho_{1 a}}{1-a}+\frac{m_{s} \xi^{2}}{\zeta^{2}-\vartheta^{2}}\right)\right.$

$\left.\times\left(\frac{\cosh (\vartheta x)}{\cosh (\vartheta l)}-\frac{\tanh (\vartheta l)}{\vartheta l}\right)-\frac{m_{s} \xi^{2}}{\zeta^{2}-\vartheta^{2}}\left(\frac{\cosh (\zeta x)}{\cosh (\zeta l)}-\frac{\tanh (\zeta l)}{\zeta l}\right)\right]$.

$\sigma_{z z}(x)=\sigma_{y y}(x)-\sigma_{a}, \quad \sigma_{x x}(x)=0$.

where

$m_{s}=\left(\rho_{0 a}-\rho_{0 *}\right) \frac{\vartheta^{2}-\xi^{2}}{\xi^{2}} \frac{D}{1-D}, \zeta=\sqrt{1-a} \xi$.

For non-homogeneous layer the skeleton density $\rho_{0}$ over the layer is shown in Fig. 3 for different layer thicknesses $(1-$ thin film, 3 - relatively thick layer).

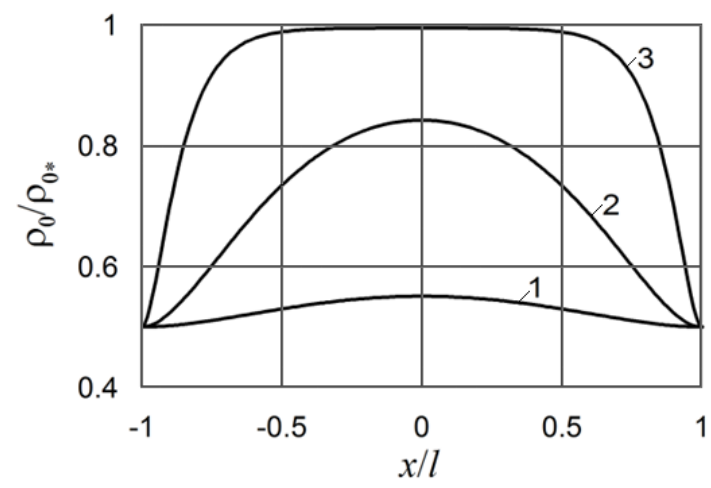

Fig. 3. Skeleton density in the layer for various layer thicknesses (the curves 1,2,3 correspond to $\xi l=2,5,20$ ) 
The increase in parameter $a$ reduces characteristic size $\zeta$, and also increases density (of skeleton) in inner areas of the body. This is shown in Fig. 4 that illustrates the value of $\varrho_{0} / \varrho_{0 *}$ at $x=0$.

The admixture density $\varrho_{1} / \varrho_{0 *}$ in the layer is shown in Fig.5.

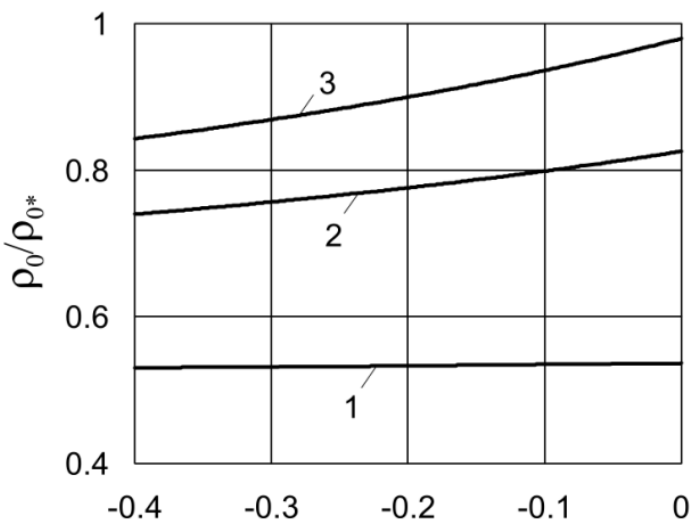

Fig. 4. Skeleton density in the middle of the layer vs. parameter $a$ for $\xi l=2 ; 5 ; 20$ (lines $1-3$ respectively), $\varrho_{a 0} / \varrho_{0 *}=0.5$ $\varrho_{a 1} / \varrho_{0 *}=0.02, \xi / \vartheta=0.5$

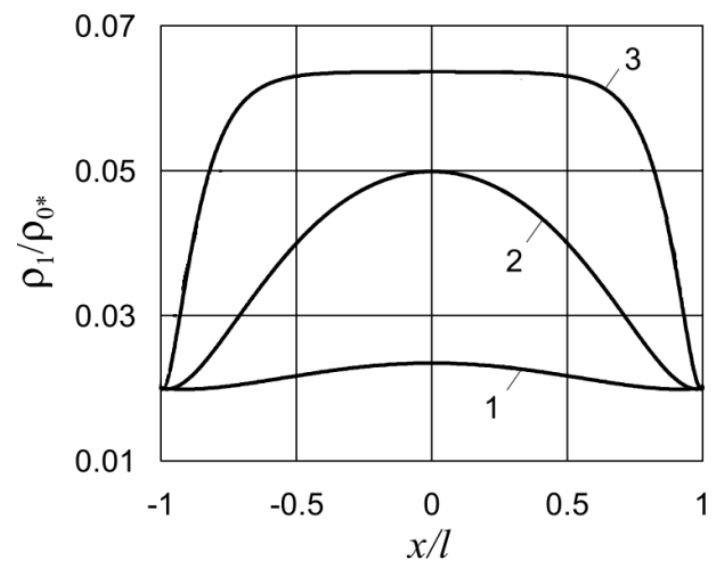

Fig. 5. Admixture density in the layer for parameters $\varrho_{a 0} / \varrho_{0 *}=0.5$, $\varrho_{a 1} / \varrho_{0 *}=0.02, a=-0.01, \xi / \vartheta=0.5$.

Curves $1-3$ correspond to $\xi l=2 ; 5 ; 20$ respectively

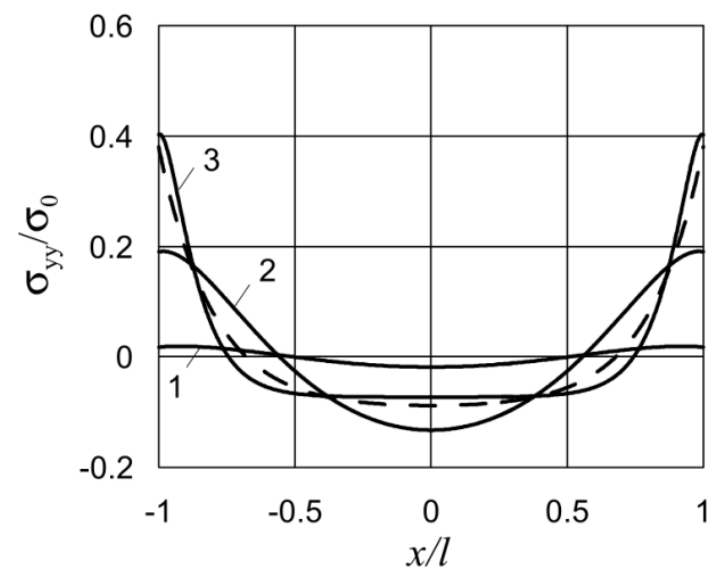

Fig. 6. Stress $\sigma_{y y} / \sigma_{0}\left(\sigma_{0}=a_{0} \rho_{0 *}\right)$ in the layer for $\xi l=2 ; 5 ; 20$ (curves 1-3), $\varrho_{a 0} / \varrho_{0 *}=0.5, \varrho_{a 1} / \varrho_{0 *}=0.02, a=-0.01$, $a_{1} / a_{0}=0.1, \xi / \vartheta=0.5$. The dash line is the stress in the absence of mass source for $\xi l=5$

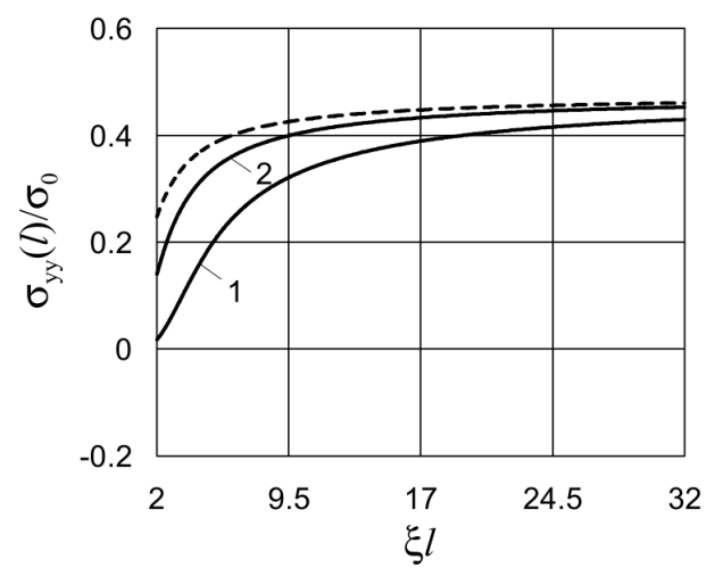

Fig. 7. Surface stress in the free layer vs. its size for $\varrho_{a 0} / \varrho_{0 *}=0.5$, $\varrho_{a 1} / \varrho_{0 *}=0.02, a=-0.01, a_{1} / a_{0}=0.1$. The dash line corresponds to the absence of mass source. The curve 1 is the stress for $\xi^{-1}=2 \vartheta^{-1}$ (the characteristic size of material heterogeneity is twice of the characteristic size of surface roughness), the curve 2 stands for $2 \xi^{-1}=\vartheta^{-1}$

In a free layer the stresses $\sigma_{y y}=\sigma_{z z}$ at external surfaces $x= \pm l$ are stretching (positive). When away from these surfaces in the depth of the layer the stresses decrease and turn into compressive near a neighborhood of the middle surface $x=0$. Taking into account mass sources comparing with the model presented in Bozhenko (2016) changes the value of the stresses and the picture of their distribution. Their impact is particularly significant in the films, which are characterized by size $\zeta^{-1}$ which is comparable to the layer (thin film) thickness. This is illustrated in Fig.6.

As can be seen from Fig. 6 the internal stresses are inherent to the free layer and the largest stresses are at the layer surfaces. They are described by formula

$\sigma_{y y}( \pm l)=\sigma_{z z}( \pm l)=\left(a a_{1}-a_{0}\right)\left[\frac{\varrho_{0 a}-\varrho_{0 *}+\varrho_{1 a}}{1-a}\right.$

$\left.\times\left(1-\frac{\tanh (\vartheta l)}{\vartheta l}\right)+\frac{m_{S} \xi^{2}}{\zeta^{2}-\vartheta^{2}}\left(\frac{\tanh (\zeta l)}{\zeta l}-\frac{\tanh (\vartheta l)}{\vartheta l}\right)\right]$.

They depend on layer size $l$, i.e. show size effect as illustrated in Fig.7.

\subsection{Admixture influence on size effect of layer strength}

The maximum stresses are important when investigating the strength. When calculating the strength parameters both external load force and internal stress should be considered. In the considered case the last is caused by structural heterogeneity of material and geometrical non-uniformity of real body surface. Using the arguments presented in the paper by Nahirnyj et al. (2015) concerning the strength for the intensity of the power load, which results in the fracture of the layer, based on (29) we write the formula

$\sigma_{a}^{c r}=\sigma_{p}-\left(a a_{1}-a_{0}\right)\left[\left(\frac{\varrho_{0 a}-\varrho_{0 *}+\varrho_{1 a}}{1-a}+\frac{m_{s} \xi^{2}}{\zeta^{2}-\vartheta^{2}}\right)\right.$

$\left.\times\left(1-\frac{\tanh (\vartheta l)}{\vartheta l}\right)+\frac{m_{s} \xi^{2}}{\zeta^{2}-\vartheta^{2}}\left(\frac{\tanh (\zeta l)}{\zeta l}-\frac{\tanh (\vartheta l)}{\vartheta l}\right)\right]$.

We denote with $\sigma_{+}$the intensity of power load that causes fracture of thick layer without admixture and rewrite the obtained formula as 
$\sigma_{a}^{c r}=\sigma_{+}-a_{0}\left(\varrho_{0 a}-\varrho_{0 *}\right)-\left(a a_{1}-a_{0}\right)\left[\frac{\varrho_{0 a}-\varrho_{0 *}+\varrho_{1 a}}{1-a}\right.$

$\left.\times\left(1-\frac{\tanh (\vartheta l)}{\vartheta l}\right)+m_{s} \frac{\xi^{2}}{\zeta^{2}-\vartheta^{2}}\left(\frac{\tanh (\zeta l)}{\zeta l}-\frac{\tanh (\vartheta l)}{\vartheta l}\right)\right]$.

In Fig.8 there is shown dependence of $\sigma_{c r}=\sigma_{a}^{c r} /\left(a_{0} \varrho_{0 *}\right)$ on layer thickness. Note that the curves 1,2 correspond to different roughnesses of the body real surface. Effect of roughness on solids strength is well known from experimental investigations.

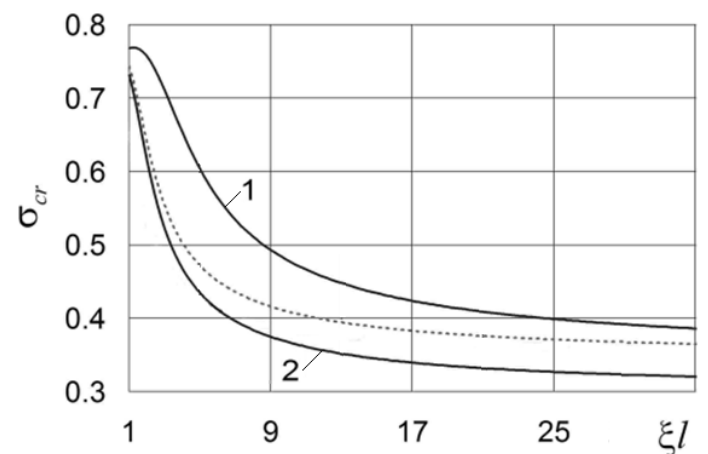

Fig. 8. Size effect of strength of the layer with admixture for $a=-0.1$, $\sigma_{+} /\left(a_{0} \varrho_{0 *}\right)=0.3, \varrho_{a 0} / \varrho_{0 *}=0.5, \varrho_{a 1} / \varrho_{0 *}=0.02$, $a_{1} / a_{0}=0.1, \xi / \vartheta=0.5 ; 2$ (curves 1,2 respectively). The dotted line shows stresses in the absence of mass source. The line 1 shows stresses for $\xi^{-1}=2 \vartheta^{-1}$ (the characteristic size of material heterogeneity is twice of the characteristic size of surface roughness), the line 2 stands for $2 \xi^{-1}=\vartheta^{-1}$

Based on the comparison of the results with the results for the body without admixture it can be argued that the presence of admixture does not change qualitatively the dependence of critical load $\sigma_{a}^{c r}$ on characteristic size of the layer (its thickness) but changes the absolute value of $\sigma_{a}^{c r}$.

\section{RESULTS AND CONCLUSSION}

The presented mathematical model of two-component thermoelastic non-homogeneous body describes multiscale size effects of mechanical properties. It features two characteristic sizes: the first one is related to heterogeneity of the body material and the second one depends on surface non-uniformity.

The presence of an admixture may change the characteristic size related to structural heterogeneity of the body material.

The equation systems within considered model allow describing bodies of both porous and nanoporous materials. It is done from unified point of view in consequence of using the methods of irreversible thermodynamics.

The introduced mass sources allow surface roughness description and porosity modeling in the real bodies compared to ideal elastic body.

The presence of the admixture in the stretched layer may cause either increase or decrease of the intensity of force load that evokes the layer fracture depending on the ratio of admixture and skeleton parameters.

The size effects of Young's modulus and Poisson's ratio are associated with local nonlinearity in the state equations of the model. The models of considered approach may be used to investigate effects observed in porous nanomaterials.

\section{REFERENCES}

1. Abeyaratne R., Knowles J.K. (1991), Kinetic relations and the propagation of phase boundaries in solids, Archive for Rational Mechanics and Analysis, 114(2), 119-154.

2. Aifantis E.C. $(2011 \mathrm{~b})$, On the gradient approach-relation to Eringen's nonlocal theory, International Journal of Engineering Science, 49(12), 1367-1377.

3. Aifantis E.C. (2011a), Gradient nanomechanics: applications to deformation, fracture, and diffusion in nanopolycrystals, Metallurgical and Materials Transactions A, 42(10), 2985.

4. Bao Y., Wen T., Samia A.C.S., Khandhar A., Krishnan K.M. (2016), Magnetic nanoparticles: material engineering and emerging applications in lithography and biomedicine, Journal of Materials Science, 51(1), 513-553

5. Berezovski A., Engelbrecht J., Maugin G.A. (2007), Front dynamics in inhomogeneous solids. Proc. Estonian Acad. Sci. Phys. Math., 56(2), 155-161.

6. Bhattacharya A., Calmidi V.V., Mahajan R.L. (2002), Thermophysical properties of high porosity metal foams, International Journal of Heat and Mass Transfer, 45(5), 1017-1031.

7. Biot M.A. (1941), General theory of three dimensional consolidation, Journal of Applied Physics, 12, 155-164.

8. Bozhenko B., Nahirnyj T., Tchervinka K. (2016), To modeling admixtures influence on the size effects in a thin film, Mathematical Modeling and Computing, 3(1), 12-22.

9. Burak Y.I., Nagirnyi T. (1992), Mathematical modeling of local gradient processes in inertial thermomechanical systems, International applied mechanics 28(12), 775-793.

10. Charalambakis N. (2010), Homogenization techniques and micromechanics. A survey and perspectives, Applied Mechanics Reviews, 63(3), 030803.

11. Coussy O. (2004), Poromechanics, John Wiley \& Sons.

12. Dönmez A., Bažant Z.P. (2017), Size effect on punching strength of reinforced concrete slabs with and without shear reinforcement, $\mathrm{ACl}$ Structural Journal, 114(4), 875.

13. Elliott J.A. (2011), Novel approaches to multiscale modelling in materials science, International Materials Reviews, 56(4), 207-225.

14. Eringen A.C. (2002), Nonlocal continuum field theories, Springer Science \& Business Media.

15. Eringen A.C., Edelen D.G.B. (1972), On nonlocal elasticity, International Journal of Engineering Science, 10(3), 233-248.

16. Geers M.G., Kouznetsova V., Brekelmans W.M. (2002), Multi-scale constitutive modelling of heterogeneous materials with a gradient-enhanced computational homogenization scheme, International Journal for Numerical Methods in Engineering, 54(8), 1235-1260.

17. Geers M.G.D., De Borst R., Peerlings R.H.J., Brekelmans W.A.M. (2001), A critical comparison of nonlocal and gradient-enhanced softening continua, International Journal of Solids and Structures, 38(44), 7723-7746.

18. Hu H., Onyebueke L., Abatan A. (2010), Characterizing and modeling mechanical properties of nanocomposites-review and evaluation, Journal of Minerals and Materials Characterization and Engineering, 9(04), 275.

19. Kachanov M., Sevostianov I. (2018), Quantitative Characterization of Microstructures in the Context of Effective Properties, In Micromechanics of Materials, with Applications (pp. 89-126), Springer, Cham.

20. Kalamkarov A.L., Andrianov I.V., Danishevsâ V.V. (2009), Asymptotic homogenization of composite materials and structures, Applied Mechanics Reviews, 62(3), 030802.

21. Markov K.Z. (2000), Elementary micromechanics of heterogeneous media, In Heterogeneous Media (pp. 1-162), Birkhäuser, Boston, MA.

22. Maugin G.A. (1979), Nonlocal theories or gradient-type theories-a matter of convenience, Archiv of Mechanics, Archiwum Mechaniki Stosowanej, 31, 15-26. 
23. Nahirnyj T., Tchervinka K. (2012), Thermodynamical models and methods of thermomechanics taking into account nearsurface and structural nonhomogeneity. Bases of nanomechanics I, Spolom, Lviv (In Ukrainian).

24. Nahirnyj T., Tchervinka K. (2013), Structural inhomogeneity and size effects in thermoelastic solids, J. Coupled Syst. Multiscale Dyn., 1, 216-223.

25. Nahirnyj T., Tchervinka K. (2014), Basics of mechanics of local non-homogeneous elastic bodies. Bases of nanomechanics II, Rastr-7, Lviv (In Ukrainian).

26. Nahirnyj T., Tchervinka K. (2015), Mathematical Modeling of Structural and Near-Surface Non-Homogeneities in Thermoelastic Thin Films, International Journal of Engineering Science, 91, 49-62.

27. Pindera M.J., Khatam H., Drago A.S., Bansal Y. (2009), Micromechanics of spatially uniform heterogeneous media: a critical review and emerging approaches, Composites Part B: Engineering, 40(5), 349-378.

28. Polizzotto C. (2003), Unified thermodynamic framework for nonlocal / gradient continuum theories, European Journal of Mechanics-A / Solids, 22(5), 651-668.

29. Rabotnov Yu.N. (1980), Elements of Hereditary Solid Mechanics, Mir Publ. Moscow (in Russian).
30. Rafii-Tabar H., Ghavanloo E., Fazelzadeh S.A. (2016), Nonlocal continuum-based modeling of mechanical characteristics of nanoscopic structures, Physics Reports, 638, 1-97.

31. Rosakis P., Knowles J.K. (1997), Unstable kinetic relations and the dynamics of solid-solid phase transitions, Journal of the Mechanics and Physics of Solids, 45(11), 2055-2081.

32. Tappan B.C., Steiner S.A., Luther E.P. (2010), Nanoporous metal foams, Angewandte Chemie International Edition, 49(27), 45444565.

33. Vafai K. (2015), Handbook of porous media, Crc Press.

34. Wang Y.M., Ma E. (2009), Mechanical properties of bulk nanostructured metals, Bulk Nanostructured Materials, 423-453.

35. Woźniak C. (1987), A nonstandard method of modelling of thermoelastic periodic composites, International Journal of Engineering Science, 25(5), 483-498.

36. Young R., Kinloch I.A., Gong L., Novoselov K.S. (2012), The mechanics of graphene nanocomposites: a review, Composites Science and Technology, 72(12), 1459-1476. 